\title{
Mathematical Modeling and Computer Simulation of Fire Phenomena
}

\author{
H. R. BAUM, K. B. McGRATTAN and R. G. Rehm \\ National Institute of Standards and Technology \\ Gaithersburg, Maryland 20899
}

\begin{abstract}
A method of studying the large scale transport of smoke and hot gases induced by fires in enclosures is described. The approach is based on solving the governing equations directly (if approximately) by decomposing the fire-driven flow field into large scale convective and small scale combustion components. In this work, results involving large scale convective transport generated by flow fields associated with typical fire scenarios are presented. The large scale flow is studied using finite difference techniques to solve large eddy simulations of the Navier-Stokes equations at high Reynolds numbers. No empirical turbulence models are employed. The basic theory behind the methodology is outlined and sample results of both large and small scale phenomena are presented and compared with related experiments.
\end{abstract}

KEY WORDS: Buoyant Convection, Computational Fluid Dynamics, Enclosure Fires, FireInduced Flows

\section{INTRODUCTION}

There exists a variety of approaches to the simulation of fires, particularly those that occur in enclosures. One way of classifying them is according to the spatial and temporal resolving power of the method employed. Thus, "zone models" resolve convective transport of smoke and hot gases in a multi-room building at the level of an individual room or passageway in the structure, with a minimum temporal resolution on the order of several seconds. Examples of this approach have been developed by Mitler and Rockett [1] for a single volume and by Tanaka [2] for multi-room configurations. Conventional feld models using $k-\epsilon$ representations of turbulence resolve such processes at smaller scales associated with the geometry of an individual room. The temporal resolution is effectively unchanged due to the need for some 
kind of averaging process inherent in such models. These models have evolved to the point where combustion and thermal radiation can be incorporated into three-dimensional simulations of fires in complex geometries. A good example of the capabilities currently available for fire simulations may be found in the computer code JASMINE developed by Cox and his collaborators [3], [4]. The price paid for this capability is a heavy reliance on empirical parameters for the description of many of these processes, together with an increase of several orders of magnitude in computational complexity to study the same scenarios accessible to zone models.

The approach outlined below, by contrast, seeks approximate solutions to the underlying Navier-Stokes equations directly. The buoyancy induced convection is computed using large eddy solutions to these equations driven by a prescribed heat release generated by the fire. Since the flows of interest have Reynolds numbers on the order of $10^{5}$, the combustion processes themselves are subgrid phenomena on these scales, and may be introduced into the model in a variety of ways. In the present work, the gaseous fuel is discretized into a large number of Lagrangian elements which burn while being convected by the large scale motion. At present, radiative transport is not explicitly included in the model. The heat release associated with each Lagrangian element may be considered as the chemical energy minus that fraction radiated out of the domain of interest.

The computer resources required are much larger than those associated with $k-\epsilon$ calculations. Indeed, they are sufficiently large to preclude simulations of many fires describable by that methodology. The current status of the large eddy simulations used to describe the convective transport of smoke and hot gases permits computation of scenarios similar to those accessible to $k-\epsilon$ models ten years ago. The difference is that a turbulence model is no longer required, as will be demonstrated below. The ability to predict the large scale transport in reasonable agreement with experiments performed at Reynolds numbers typically associated with turbulent flow has important implications. It shows that most of the convective transport of mass, momentum, and energy in fire induced flows is carried in wavelengths ranging about two orders of magnitude down from those imposed by the enclosure geometry. The fine structure of the turbulence, if present, plays a relatively small role in these phenomena.

\section{MATHEMATICAL MODEL}

Since the wide range of length and time scales to be considered is primarily due to the complex nature of the fire induced flow field, the starting point for the analysis is the fact that the velocity vector $\vec{u}$ can always be decomposed into a solenoidal field $\vec{v}$ plus an irrotational flow derived from a potential, $\vec{u}=\vec{v}+\nabla \phi$, where $\nabla \times \vec{v}=\vec{\omega}$ and $\nabla \cdot \vec{v}=0$. This mathematical definition of the solenoidal component of the velocity field can be interpreted physically as a statement that the "source" of the solenoidal field is the fluid vorticity. Since most of the vorticity in a fire is generated by the thermally induced buoyancy, $\vec{v}$ represents the large scale convective transport.

Following Rehm and Baum [5], we consider the Boussinesq form of the equations of motion for the large scale convection. This limits the domain of applicability of the current simulations to regions not in or near the combustion zone of the fire. At present, we are also limited to scenarios that can be reasonably approximated as two dimensional. These limitations are 
not fundamental to our approach, but are a reflection of the computational resources currently available to us. As noted at the end of the paper, this barrier will soon disappear.

For the two dimensional case, it is convenient to write the equations of motion as two convection-diffusion equations for the density perturbation $\tilde{\rho}$ and the scalar vorticity $\omega$ in dimensionless form

$$
\begin{aligned}
& \frac{\partial \tilde{\rho}}{\partial t}+\vec{v} \cdot \nabla \tilde{\rho}=-q+\frac{1}{\operatorname{Re} \operatorname{Pr}} \nabla^{2} \tilde{\rho} \\
& \frac{\partial \omega}{\partial t}+\vec{v} \cdot \nabla \omega=\nabla \times \tilde{\rho} \frac{\vec{g}}{g}+\frac{1}{\operatorname{Re}} \nabla^{2} \omega
\end{aligned}
$$

The solenoidal velocity $\vec{v}$ is recovered from the stream function $\psi$ which is found by solving the Poisson equation

$$
\nabla^{2} \psi=-\omega
$$

The net convective heat release due to burning is represented by the term $q$. There is no explicit accounting for radiative heat transfer in the model. The density perturbation $\tilde{\rho}$ is related to the actual density $\rho$ by

$\rho=\rho_{\infty}(1+\beta \tilde{\rho})$

The small perturbation parameter $\beta$ is the Froude number, given as $\beta=V^{2} / g h$ where $h$ is the enclosure height and $V$ is the characteristic velocity which is related to the strength of the two dimensional heat source by the expression

$V=\left(q_{0} g / \rho_{\infty} c_{p} T_{\infty}\right)^{1 / 3}$

Equations (1)-(3) are a mixed parabolic/elliptic system of partial differential equations; i.e., the equations for the density and for the vorticity are parabolic, whereas that for the stream function is elliptic. To solve the equations numerically, we replace all of the spatial derivatives by second order central differences and advance the solution in time with an Alternating Direction Implicit (ADI) scheme [6]. The physical domain may be taken as any simply connected polygon, which is mapped conformally into a rectangle whose aspect ratio is dependent on the shape of the polygon.

The resolution of the computational grid determines the maximum resolvable Reynolds number for a given run. Setting the Reynolds number thus determines the range of dynamically active length scales included in the simulation. The ratio of the geometric length to the minimum resolvable scale is essentially the square root of the Reynolds number. Maximizing this ratio within the constraints of the available computing resources is the essence of the large eddy simulation. For the examples presented here, we have used grids with up to six hundred thousand grid cells, and Reynolds numbers approaching 50,000. CPU times are about $3 \times 10^{-5}$ seconds per grid cell per time step on an IBM RISC/6000 (model 550). The size of a time step varies from one application to another. For the examples to follow, several thousand time steps are required to complete a computation, requiring from 10 up to 50 hours of CPU time. 

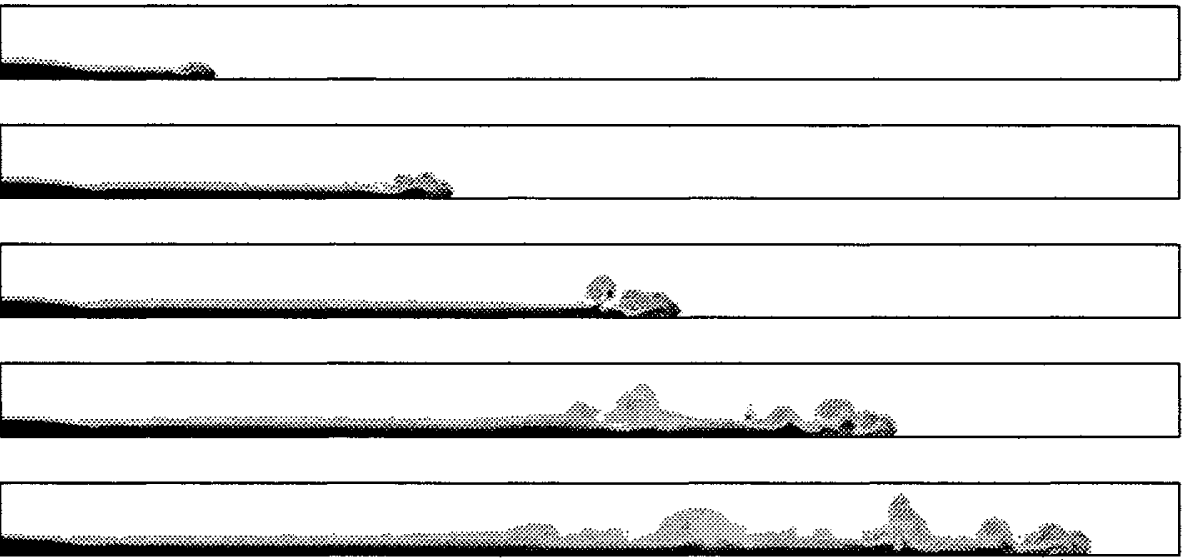

FIGURE 1: A gravity current develops as heavy fluid is pumped into the channel at the lower left. Fluid is evacuated at the upper left.

\section{RESULTS}

We wish to validate the methodology by comparing the computations with experimental results. Often the movement of smoke and hot gases in an enclosure is modeled using fresh water and salt water mixtures. Indeed, the equations of motion for the salt/fresh water mixture are exactly those given above, with the thermal conductivity analogous to the material diffusivity of the salt water, and the Prandtl number analogous to a Schmidt number. The analogy is not perfect because the Prandtl number for air is about .7, whereas the Schmidt number for salt water is on the order of several hundred. We can compute directly flows whose Schmidt numbers are on the order of one, and we can also consider the special case where the Schmidt number is infinite, solving equation (1) in Lagrangian form (with density determined by initial salinity concentration and $q \equiv 0$ )

$\frac{D \tilde{\rho}}{D t}=0$

For the sample calculations below, we have found that the results are insensitive to the size of the Prandtl/Schmidt number. This is in agreement with the experiments and analysis of Steckler [7] which have demonstrated that the salt water experiments are a quantitatively accurate analogy of smoke movement in buildings. In any event, the focus of our investigations is the latter phenomenon, and thus the infinite Schmidt number case is considered only as a means of comparing our computations with experiment.

We first look at a gravity current. Consider the flow induced by the introduction of salt water into a long rectangular tank filled with fresh water. The denser salt water flows along the bottom of the tank in much the same way that hot smoke moves along the top of a corridor of a burning building. Chan and Zukoski [8] have conducted gravity current experiments in conjunction with their research on smoke movement. They use a tank $274 \mathrm{~cm}$ long, $15 \mathrm{~cm}$ high, and $15 \mathrm{~cm}$ wide filled with fresh water. Salt water is pumped into the bottom of the tank through a slot 


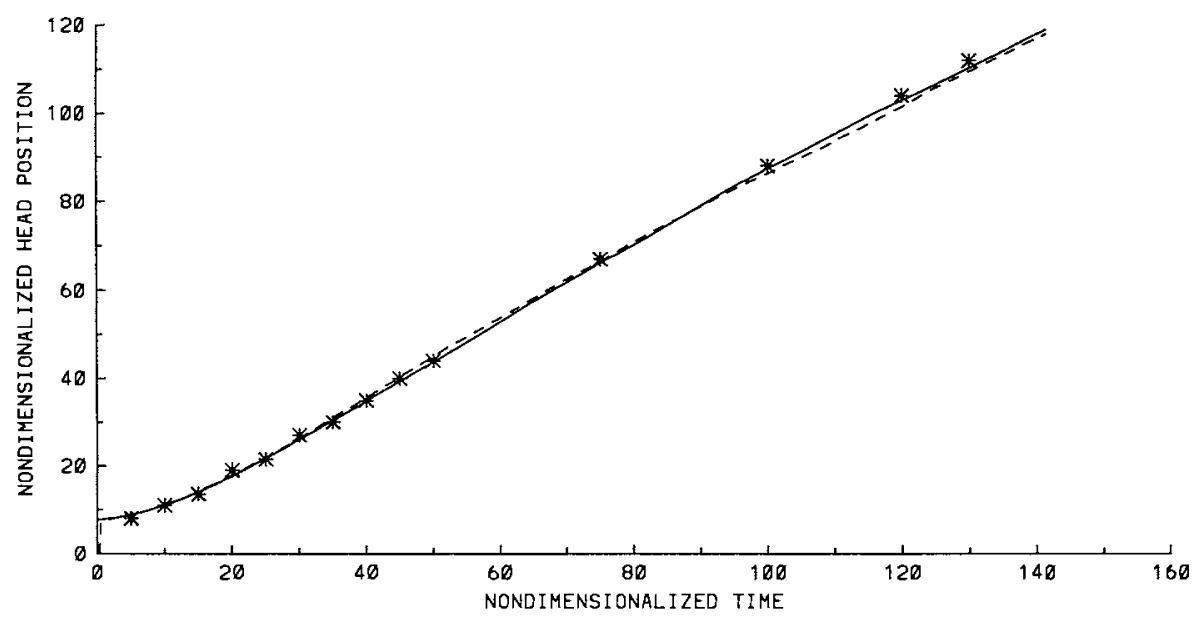

FIGURE 2: A comparison of the gravity current trajectories for a nondiffusive computation (solid line), a diffusive computation (dashed line), and a salt water experiment (stars). The length and time scales are determined by the entry height and the characteristic velocity of the current.

at a specified (constant) rate determined by the prescribed buoyancy flux and entry height of the current. For the computation, we use a $16 \times 1$ rectangular enclosure ( $3072 \times 192$ cells). Figure 1 displays a few profiles of the computed gravity current. The Reynolds number based on the entry height of the current is about 2,000 , but it is 30,000 based on the enclosure height. Figure 2 shows the excellent agreement in the speed of the experimental and the computational currents. The speed of the current is relatively unaffected by the Schmidt number.

A more challenging simulation is displayed in Figure 3. Here we are modeling the mixing of two fluids of different temperatures at an interface, which can be thought of as a window. The small enclosure to the left is initially filled with a hot fluid, while the larger area outside is at ambient temperature. When the interface between the two fluids is removed, the cold fluid pours into the compartment while the hot fluid escapes. The scenario is of interest when studying backdraft phenomena. This occurs when a fire in a room exhausts the available supply of oxygen and appears to die out, only to be explosively reignited when fresh air re-enters the room through a newly opened door or window. Computations similar to that presented in the figure have compared very well with salt water experiments conducted at the University of California, Berkeley by Fleischmann, Pagni and Williamson [9] in conjunction with their research on backdrafts.

The calculations were also compared with half-scale backdraft experiments conducted by Fleischmann as part of his study. An enclosure (1.2 m high, $1.2 \mathrm{~m}$ wide and $2.4 \mathrm{~m}$ long) with a slot opening at one end was used to study the behavior of fresh, cool currents of air streaming into a compartment filled with heated gases. Bidirectional probes were installed in the compartment opening to measure the velocity of the fluid entering and exiting. Figure 4 compares the velocities at the compartment opening with the calculated results several seconds 


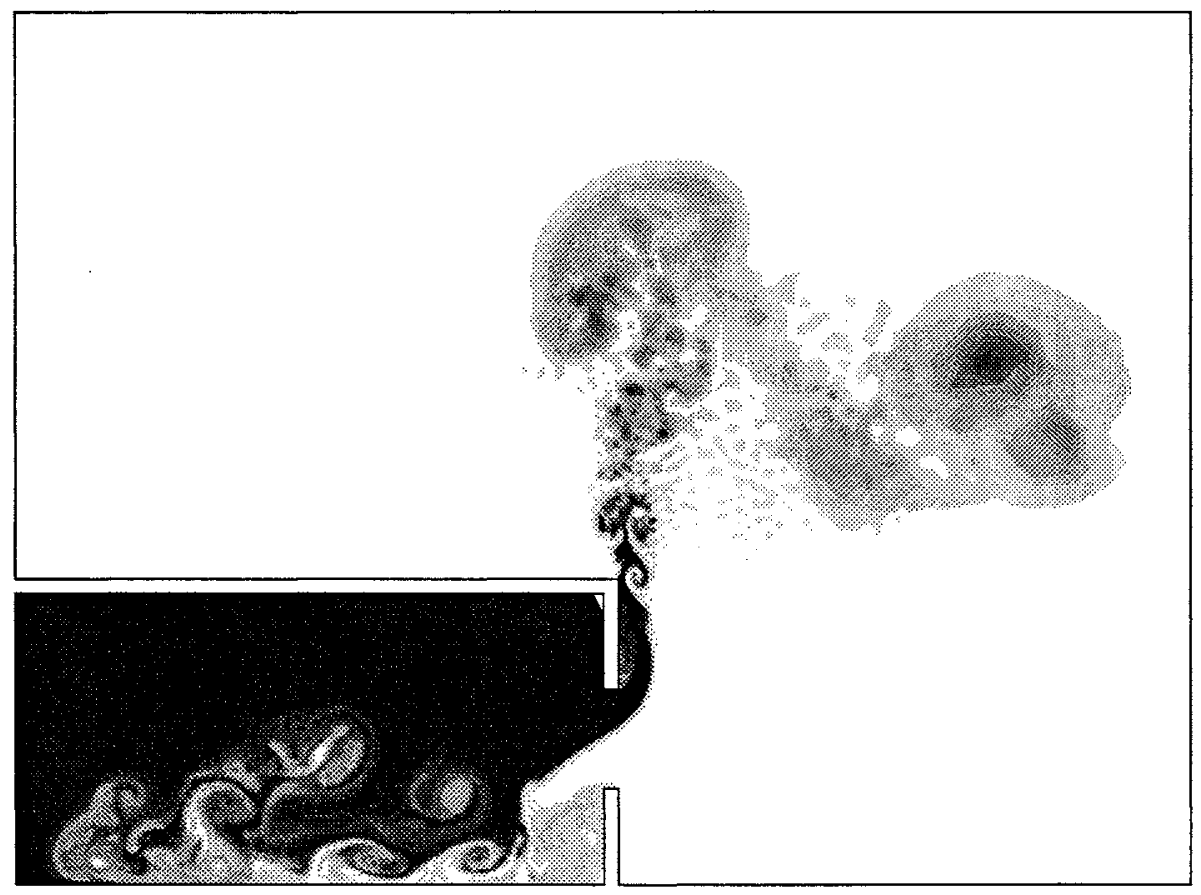

FIGURE 3: The mixing of hot (black) and fresh (white) air after the opening of a window. The grid size is $768 \times 192$ cells, and the Reynolds number is 30,000.

after the door separating the two fluids is opened. It should be noted that the computed profile is an instantaneous result, with no time averaging. No attempt to correct for any possible probe misalignment with the flow direction has been made. The temperature range in the experiment is probably beyond that for which the Boussinesq approximation is valid, and three-dimensional effects at the sides of the opening are not accounted for. Despite these difficulties, the agreement between experiment and a theory containing no adjustable parameters is quite encouraging.

Finally, we consider the flow induced by a fire in a larger enclosure that illustrates considerable geometric complexity. The geometry for the simulation contains such building elements as an atrium, a horizontal corridor, a stairwell, and a vertical shaft. Unlike the two examples above, the flow is driven by the introduction of heat-laden particles which represent the fire rather than some initial temperature difference. The value of $\beta$ is determined from the overall heat release rate and enclosure geometry; see Steckler et al. [7] for details. Our combustion model is simple - each particle is initialized with a fixed amount of energy which decays exponentially in time. The addition of the heat-laden particles is a first step towards a more comprehensive combustion model, which will include the effects of radiation and thermal expansion. The examples here demonstrate the large-scale dynamics of the solenoidal component of velocity. Figure 5 presents the temperature field of a fire simulation in which the particles originate at the base of a stairwell. The Reynolds number for the run is 10,000 based on the height of a horizontal segment of corridor and the Prandtl number is 0.7 . Upwards of 10,000 particles 


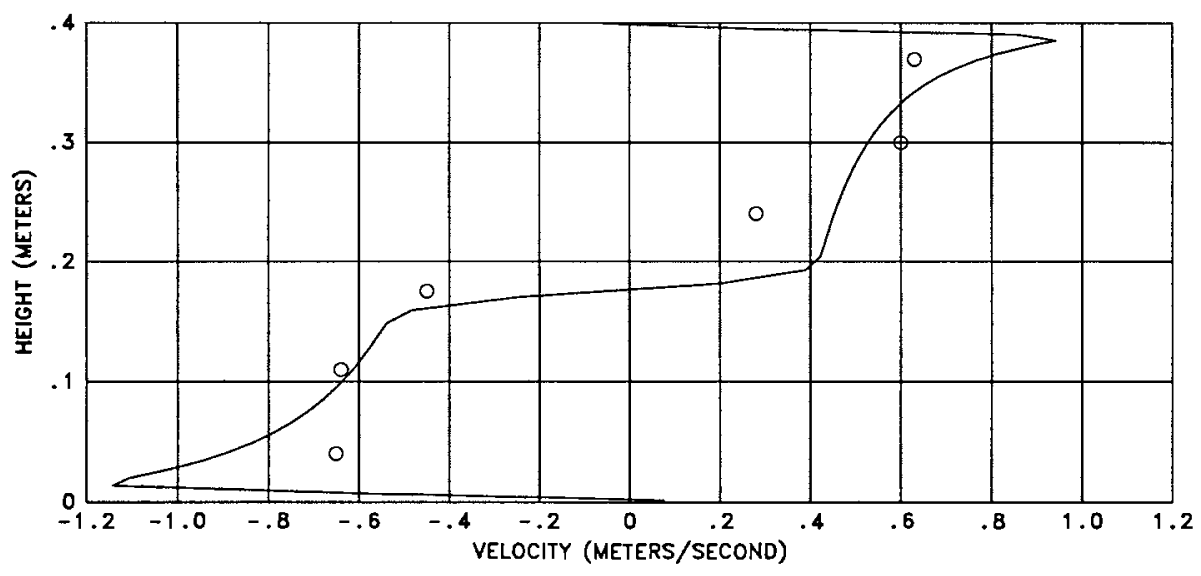

FIGURE 4: A computed velocity profile at the compartment opening shown above is compared with experimental results (circles) 4.5 seconds after the opening of the compartment. The temperature difference between the two fluids is about $90^{\circ} \mathrm{C}$.

were used to represent the source of heat. These particles are advected with the flow, and their heat is applied to the appropriate grid cells at every time step in the calculation. The interesting feature of this scenario is the concentration of smoke in the stairwell. A gravity current is formed at the base of the stairway and proceeds upwards along the ceiling of the stairway. However, the gas is cooled due to the increased entrainment caused by both the inclination of the stairwell and the reverse flow of the fluid, and it falls off the ceiling sooner than would be expected. This phenomenon has been noted by Chan et al. [8] in conjunction with their gravity current experiments.

\section{CONCLUSIONS}

The approach to the simulation of fire induced convective transport outlined above has the virtue that it is based directly on our understanding of the equations governing fluid flow. As this understanding improves, the methodology can be modified to accept the necessary changes. The advantage of decomposing the velocity field into solenoidal and irrotational components is that it allows one to make the most efficient use of the available computational resources, whether that entails high resolution 3D simulations of the buoyancy driven solenoidal flow in geometries of increasing complexity or the inclusion of radiation and more realistic combustion in more simple geometries. Microprocessor based computer systems now becoming commercially available provide sufficient computing power to allow three dimensional simulations for individual rooms at resolutions comparable to those shown above in two dimensions. Advances in both microprocessor design and parallel processing will certainly permit the simulation of smoke and hot gas transport in realistic building geometries without using turbulence models by the end of the decade. Although the mathematical modeling effort and computer resources required are much higher than in more conventional computations of these phenomena, we believe that the possibilities opened by such an approach are well worth the effort. 


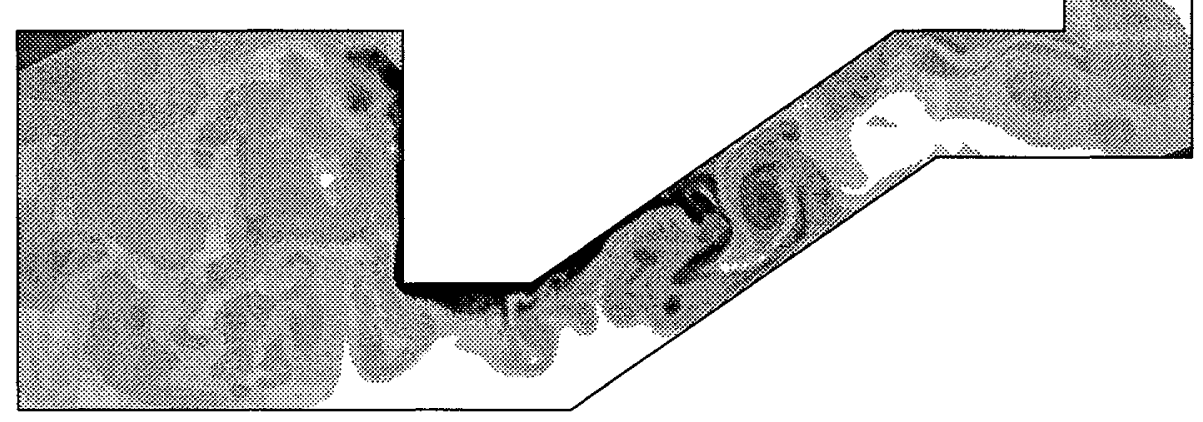

FIGURE 5: The location of smoke and hot gases in a building fire scenario. The grid size is $1536 \times 128$ cells, and the Reynolds number is 10,000 .

\section{REFERENCES}

[1] Mitler, H.E., and Rockett, J. A., "Users guide to FIRST, a comprehensive single room fire model”, NBSIR 87-3595, National Institute of Standards and Technology, Gaithersburg, Maryland, 1987.

[2] Tanaka, T., "A model of multiroom fire spread", NBSIR 83-2718, National Institute of Standards and Technology, Gaithersburg, Maryland, 1983.

[3] Markatos, N.C., Malin, M.R., and Cox, G., "Mathematical modeling of buoyancy induced smoke flow in enclosures", Int. J. Heat and Mass Transfer, 25, 63-75, 1982.

[4] Kumar, S., Gupta, A.K., and Cox, G., "Effects of Thermal Radiation on the Fluid Dynamics of Compartment Fires", in Fire Safety Science - Proceedings of the Third International Symposium, pp. 345-354, Elsevier, London, 1991.

[5] Rehm, R.G. and Baum, H.R., "The Equations of Motion for Thermally Driven, Buoyant Flows", Journal of Research of the NBS, 83, 297-308, 1978.

[6] McGrattan, K.B., Rehm, R.G., and Baum, H.R., "Fire-Driven Flows in Enclosures", J. Comp. Phys., 110:2, February 1994. 
[7] Steckler, K.D., Baum, H.R., and Quintiere, J.G., "Salt Water Modeling of Fire Induced Flows in Multicompartment Enclosures", in Twenty-First Symposium (International) on Combustion, pp. 143-150, The Combustion Institute, Pittsburgh, Pennsylvania, 1988.

[8] Chan, W.R., Zukoski, E.E., and Kubota, T., "Experimental and Numerical Studies on Two-Dimensional Gravity Currents in a Horizontal Channel", Guggenheim Jet Propulsion Center, California Institute of Technology, Pasadena, California, 1992.

[9] Fleischmann, C.M., Pagni, P.J., and Williamson, R.B., "Exploratory Backdraft Experiments", Fire Technology, in press. 
